\title{
An Overview of Cerebral Malaria: Lessons Learnt from Observations in Humans and Experimental Models
}

\author{
Sitabja Mukherjee ${ }^{1}$, Prashant Singh ${ }^{2}$ and Santosh K Kar*2 \\ ${ }^{1}$ School of Biotechnology, KIIT deemed to be University, Bhubaneswar, Odisha, India \\ ${ }^{2}$ Nano Herb Research Laboratory, KIIT Technology Business Incubator, KIIT deemed to be University, Bhubaneswar, Odisha, \\ India
}

*Corresponding author: Santosh K Kar, Nano Herb Research Laboratory, KIIT Technology Business Incubator, Kalinga Institute of Industrial Technology (Deemed University), Bhubaneswar-751024, Odisha, India

\section{ARTICLE INFO}

Received: 蔧 March 26, 2021

Published: 幽 April 08, 2021

Citation: Sitabja Mukherjee, Prashant Singh, Santosh K Kar. An Overview of Cerebral Malaria: Lessons Learnt from Observations in Humans and Experimental Models. Biomed J Sci \& Tech Res 35(1)-2021. BJSTR. MS.ID.005639.

\section{ABSTRACT}

Malaria continues to be a serious public health problem killing 409,000 people annually worldwide, half of which come from 6 countries from sub-Saharan Africa. Out of 5 different species of malaria parasites, Plasmodium falciparum infection in humans is the main cause of mortality. It induces Cerebral Malaria pathology in 10-15\% of affected individuals. Cerebral Malaria manifests as a neuropathological complication, leading to the disruption of the Blood Brain Barrier and significant neuronal damage that eventually causes coma and death in humans. Most of the clinical information about Cerebral Malaria in humans has come from postmortem examinations of the brain and therefore the use of experimental animal models became necessary. This provided valuable insight about the mechanistic processes that lead up to the induction of cerebral malaria pathology and also enabled screening and evaluation of therapeutic molecules for prevention of death. Unfortunately, till date no effective therapeutic interventions except artemisinin and quinine based therapies are available therefore understanding the different events that causes the pathology by learning from all the different observations obtained thus far, becomes of paramount importance.

Abbreviations: CM: Cerebral Malaria; WHO: World Health Organization; SM: Severe Malaria; MRI: Magnetic Resonance Imaging; MRS: Magnetic Resonance Spectroscopy; CT: Computational Tomography; PbA: Plasmodium berghei ANKA; BBB: Blood Brain Barrier; CNS: Central Nervous System; ECs: Endothelial Cells; ICAM-1: Intercellular Adhesion Molecule-1; VCAM-1: Vascular Cell Adhesion Molecule-1; EPCR: Endothelial Protein C Receptor; ECM: Experimental Cerebral Malaria; CS: Curdlan Sulfate; TNF: Tumour Necrosis Factor; BMS: $\beta$-Methasonehemisuccinate; GSK3 $\beta$ : Glycogen Synthase Kinase 3

\section{Introduction}

Malaria has been a significant threat to human health throughout history. As an effective vaccine against malaria is not available yet vector control measures like the use of insecticidetreated mosquito nets and indoor residual spraying has received significant attention to prevent and reduce malaria transmission. According to the World Malaria Report, released on 30 November 2020, there were 229 million cases of malaria in 2019 resulting in 409000 deaths annually world wide mostly affecting children under 5 years of age from 6 countries in sub-Saharan Africa [1]. Five different species of protozoan parasites belonging to the genus Plasmodium are capable of infecting humans and causing malaria
[2]. Out of these, two major species such as P. vivax and P. falciparum, are responsible for most of the morbidity and mortality in humans. The signs and symptoms that are seen in individuals who are infected and develop clinical signs of illness, varies widely between children and adults [3]. Malaria manifests itself by inducing the following types of conditions in humans - it may be accompanied by electrolyte and metabolic imbalances, respiratory failure, severe anemia, jaundice, pulmonary edema or a severe neuropathological condition termed as Cerebral Malaria (CM) [4]. The World Health Organization (WHO) defines $\mathrm{CM}$ as an unarousable coma that results from clinical manifestation due to P. falciparum infection in humans 
when it is not attributable to other causes [5]. According to hospital admission records, $1-2 \%$ of all $P$. falciparum infections result in CM, having a fatality rate of $10-20 \%$ [6]. Furthermore, at least 10 to $20 \%$ of those who survive and recover from CM suffer significant post-disease neurological sequelae for several years after cure [7]. In the regions of high malaria transmission, the age of the affected individuals and the degree of exposure to the parasite causing the infection are key determinants to the susceptibility to severe malaria. Developing complete immunity from malaria has not yet been possible for humans due to the complexity of the parasite's life cycle; however acquisition of clinical immunity, which confers protection from life-threatening illnesses are achievable. But the acquisition of clinical immunity requires numerous exposure to the parasite during an individual's life span through infective mosquito bites [8]. Most children, who get repeatedly exposed to malaria in areas of high transmission, acquire some form of resistance against the severe forms of the disease, provided they manage to survive the first few years of their lives after getting infected [8]. In areas of low transmission however, Severe Malaria (SM) occurs equivalently in all age groups, and is slightly more common in adults. This is because clinical immunity to malaria does not occur at all or takes a very long time to acquire and may persist for a short period. Although adults generally have a fairly good resistance against developing SM, infants are at an increased risk of developing malignant malaria, especially during their first years of life. However, the older children who've had atleast a single prior exposure to $P$. falciparum infection, are disproportionately at risk of developing CM symptoms [9]. The epidemiology of CM is suggestive of the fact that the predisposition to disease manifestation or its prevention is ultimately determined not only by the virulence of the invading parasite; but is also attributable to the host's own immune response which contributes to the degree of disease pathogenesis and outcome [10-12].

\section{Clinical Attributes of the Pathology Observed in CM Patients}

Post-mortem examination of human brain have helped to uncover the extent and type of brain pathology that occurs due to CM. leading to death. The prevalance of haemorrhages and swelling in the white matter of the subcortical rim and corpus callosum as well as presence of petechial and ring haemorrhages in cerebral and cerebellar cortices have been some of the most commonly reported findings from such examinations [13]. Histopathological analyses of brain of CM patients after death has revealed the presence of parasitized erythrocytes clogging the cerebral capillaries [13]. The pathologic features of CM also include the presence of monocytes and macrophages within cerebral vessels together with the sequestration of pigmented macrophages and parasitized erythrocytes confined to cerebral blood vessels [14]. Due to the lack of detailed comparative histopathological analysis, it is extremely difficult to determine the variations in $\mathrm{CM}$ pathology between children and non-immune adults, but given the number of differences in the symptoms of pediatric and adult $\mathrm{CM}$ it is plausible that there may be some age-related variations in the cerebral pathology observed in these groups [15]. Although parasite sequestration,haemorrhages and inflammation are some of the common findings in histological analyses of the brains of the majority of CM patients, it is quite probable that CM is not a homogenous syndrome. In fact, three distinct and different patterns of histopathological changes have been observed in African children. Firstly, there is the 'classical' pattern with parasite sequestration, perivascular haemorrhages and immune cell infiltration observed within brain micro-vessels due to CM. Next, there are incidences where parasite sequestration has been observed within the brain, but without any of the other aforementioned abnormalities. And lastly, numerous cases have been observed where individuals with high peripheral parasitemia develop a syndrome that is clinically defined as CM but where there has been no evidence of parasite sequestration within the brain $[16,17]$. The reasons for the observed variations in pathology are not clear and are hard to elucidate with utmost clarity but investigations indicate that it may be attributable to genetic variations in either the hosts or the parasites or may be combination of both, along with external environmental factors and the differences in the intensity of the immune response mounted against the parasite to control the infection.

\section{The Limitations of Studies of Human Cerebral Malaria \& Importance of Experimental Models in Animals}

Cerebral Malaria arises as a result of a complex sequence of inter-related events that ultimately lead to the manifestations of the clinical symptoms observed in affected individuals. The sequestration of trophozoite infected Red Blood Cells (pRBC) in the small blood vessels together with the rupture of infected red blood cells and the release of parasite-derived toxins could all be the defining features that lead to the induction of the pathology associated with the development of CM $[18,19]$. The effects of systemic and local events including pRBC sequestration and breach, the activation of monocyte, endothelial, and glial cells, the release of harmful inflammatory substances, and the exact sequence of events with their individual timing that ultimately lead up to the disease manifestation are very much unknown when it comes to precipitating the pathogenesis of CM. Since the histopathological analysis of brains of CM patients can only be done after death during post mortem, it is extremely difficult to describe the factors that would have lead to the onset of the disease or the determinants for its abrogation when treatment would have been successful. Such investigations and comparisons are essential to delineate truly pathogenic systemic and intra-cerebral processes from neutral and/or protective responses. Increased utility of noninvasive in vivo imaging techniques, such as Magnetic Resonance Imaging (MRI) and spectroscopy (MRS) and Computational Tomography (CT), should hopefully help to address these issues, 
but these studies are severely restricted by ethical constraints and the availability of the expensive specialized equipment in malariaendemic areas [20-22]. In order to observe and understand the immunological pathways that are primarily elicited to control the infection together with the manipulations brought about at the level of the invading pathogen for its own survival and persistence, it is imperative to implement direct intervention studies and move beyond purely descriptive conjectures, which is an extremely difficult undertaking. Examination of the peripheral blood often provides very limited information about the immunological and parasitological environment in the brain as the peripheral blood parasitaemia does not accurately reflect total parasite biomass. Total parasite biomass is a stronger correlate of severe malarial disease than the peripheral parasitaemia [23]. The situation is made further complicated as the patients usually present themselves to the hospitals only when the disease has reached a very advanced stage. It is clear that other approaches - in combination with human studies - are required to fully understand the pathogenesis of CM. Much of our understanding of mammalian physiology has come from studies of animals and the extent of the conservation of basic immunological and neuropathological processes between laboratory rodents and humans has become more apparent $[24,25]$. Primate models of CM, including P. knowlesi and P. coatneyi infections in Rhesus monkeys and $P$. falciparum infection in squirrel monkeys [26,27], have allowed the investigation of some aspects of $\mathrm{CM}$, but these models are prohibitively expensive and are done with smaller number of animals due to ethical reasons. neuropathological syndromes have been shown to develop in certain strains of inbred mice infected with various strains of Plasmodium berghei $[28,29]$. There has been and continues to be significant disagreement within the malaria research community as to whether the murine models share sufficient similarities with human cerebral malaria to make them relevant or useful. However, experimental models have proven invaluable for understanding the pathogenesis of numerous autoimmune and infectious diseases of humans and many vaccines and immuno-therapies currently in use were initially developed and tested in experimental models [25]. Therefore from that aspect, the use of relevant experimental animal models has significantly aided in the study of CM and are indispensable tools for gaining valuable insight and improving our understanding of the disease pathogenesis.

\section{Plasmodium Yoelii XL and Plasmodium berghei K173}

Although more extensively studied as a model of hyperparasitaemia and failure of parasite control, PyXL has been shown to sequester within the brain microvasculature and produce a cerebral syndrome in mice comparable with human cerebral malaria. [30-32]; however, the hyper-parasitaemia associated with this infection (rapidly ascending peripheral parasitaemia that can reach $80-100 \%$ ) is not typical of human CM cases and this model is not widely used to study CM. In a few studies, P. berghei K173 has been found to induce CM-like symptoms, but the dosedependent onset of Experimental Cerebral Malaria (ECM) in this model (inducing cerebral pathology after low dose infection which does not happen when a high dose is used) also limits its utility as a model of human CM. P. berghei K173 parasite is frequently used to induce a non-ECM-infection to compare with the most widely used model of ECM involving P. berghei ANKA infection [33,34].

\section{Plasmodium berghei ANKA}

The Plasmodium berghei ANKA $(\mathrm{PbA})$ model manifests many events seen during human $\mathrm{CM}$ and is accepted as the best available experimental mouse model of cerebral malaria. Infection of susceptible strains of mice, including C57BL/6 or CBA mice, leads to the development of fatal cerebral pathology, with clinical signs including ataxia, fitting, respiratory distress and coma [35,36]. The time to onset of clinical signs varies depending on the dose of parasite used for causing infection, the genetic background of the host and the specific clone of the infecting parasites, but is typically between 5 to 10 days post-infection [36]. As in humans, there is a rapid deterioration in the condition of infected animals once clinical symptoms set in, with death often occurring within 4 or $5 \mathrm{hr}$ after the onset of neurological signs. Multiple areas of bloodbrain barrier disruption with vascular leakage involving the cortex, cerebellum and olfactory bulb are observed in brains of PbAinfected mice displaying signs of ECM, with loss of specific neuronal cell populations within the cortex and striatum accumulation of pRBC within blood vessels and focal perivascular inflammation [37-39]. ECM is also associated with the significant accumulation of platelets within the brain vasculature [40]: platelets have been shown to directly promote endothelial cell damage during infection. Cognitive dysfunction in mice during P. berghei ANKA infection, as shown by impaired memory, is directly correlated with haemorrhage and inflammation, including microglial activation [41]. Indeed, the accumulation of monocytes and macrophages, and activation of brain resident mononuclear cells, including astrocytes and microglial cells is believed to be a key feature of ECM. As in humans, genetic and environmental factors determine the susceptibility of mice to ECM. For example, the resistance of F1 intercrossed BALB/c (resistant) and C57BL/6 (susceptible) mice to the development of ECM is determined by age, and environmental exposure, with young mice (8-10 weeks) susceptible to ECM and older mice (16-20 weeks) resistant to the development of cerebral pathology [41].

\section{The Involvement of Blood Brain Barrier in CM Pathology}

Several studies on patients in Africa and Asia have shown that the disruption of the Blood Brain Barrier (BBB) in CM leads to severe neurological complications including 
1. Intracerebral hemorrhage,

2. Seizures resulting from electrolyte imbalance, and

3. Increase in intracranial pressure due to widespread edema resulting in axonal damage [42-45].

All of these factors ultimately result in Central Nervous System (CNS) dysfunction and death. The BBB is a semipermeable membrane that separates the peripheral blood from the brain parenchyma. The BBB comprises a monolayer of Endothelial Cells (ECs) joined together by tight junctions and the underlying basal lamina. The integrity of the BBB is further supported by pericytes and the astrocyte end-feet [46]. The BBB, together with microglia and neurons, forms the neurovascular unit. Apart from serving as an anatomical barrier, the BBB plays a crucial role in the homeostasis of the CNS by facilitating the transport of nutrients such as glucose and amino acids from the blood to the CNS and removal of metabolic waste products from the CNS into the blood by means of specific transport channels [46]. Up to now, the precise underlying mechanisms leading to the disruption of BBB integrity during CM have remained unclear. Several mutually nonexclusive events have been associated with BBB breakage in human and murine studies, namely

1. Sequestration of iRBCs to the brain ECs [47-49],

2. An excessive inflammation resulting from heightened intracerebral proinflammatory cytokine response [49],

3. Disseminated intravascular coagulation in the brain $[50,51]$, and

4. Dysregulation of vascular ECs [52]. In addition, a growing number of reports implicate two other major factors in the disturbance of the BBB:

a) The host's pathway for tryptophan metabolism, also known as the kynurenine pathway, and

b) Extracellular vesicles released by the host and the parasite called microparticles.

Accumulation of parasitized erythrocytes to the luminal site of microvessel ECs triggers CM. The P. falciparum infected erythrocyte membrane protein-1 (PfEMP1) facilitates the adhesion of the infected erythrocytes to the brain vascular ECs. PfEMP1 is localized to small cup-like protrusions on the plasma membrane of infected erythrocytes called knobs, which mediate the binding of the iRBCs to the ECs [53]. PfEMP1 binds to various receptors on the brain ECs, including the intercellular adhesion molecule-1 (ICAM-1), the vascular cell adhesion molecule-1 (VCAM-1), and the cytokine-activated Endothelial Protein C Receptor (EPCR), thereby immobilizing the iRBCs on the vascular endothelium [54-56]. Patients with CM show an expansion of parasites expressing PfEMP1 that bind to both ICAM-1 and EPCR $[57,58]$. Platelets induce the adhesion of iRBCs to one another, forming large autoagglutinates [59]. Furthermore, noninfected erythrocytes form rosettes around the iRBCs, contributing further to microvascular obstruction [59]. Since sequestration of iRBCs to the brain endothelium plays a critical role in the development of $\mathrm{CM}$, prevention of sequestration and desequestration of the iRBCs are attractive therapeutic approaches, discussed in detail by Glennon, et al. [60]. The binding of PfEMP1 to the receptors on the ECs may trigger multiple signaling pathways in ECs, resulting in the reorganization of tight-junction complexes, ultimately leading to the leakiness of the BBB. In vitro studies on transendothelial migration of leukocytes, using human and murine ECs, have shown that engagement of ICAM-1 induces the phosphorylation of cytoskeleton-associated proteins, including FAK, paxillin, p130Cas, and cortactin, resulting in remodeling of the endothelial cytoskeleton to facilitate BBB opening [61].

\section{The Role of the Host's Immune Response in the Pathogenesis of CM}

Human Cerebral Malaria: The aetiology of CM provides clear evidence for the involvement of the host immune response on the basis of a distinct pattern of cytokine profiles that are observed in individuals with CM. A high concentration of plasma TNF, IFN- $\gamma$, IL-6 along with elevated ratios of pro-to anti-inflammatory cytokines are consistently observed in CM patients. High concentrations of inflammatory cytokines in the cerebrospinal fluid have been associated with a high risk of developing neurological sequelae [62]. Investigations have demonstrated that binding of pRBCs to brain endothelial cells, causes the activation of the NF-kB pathway, leading to the production of CCL20, CXCL1, CXCL2, IL-6 and IL-8 [63]. How these inflammatory responses lead to the onset of CM are still topics for discussion. It has been observed that the induction of high concentrations of pro-inflammatory cytokines lead to the upregulation of endothelial ICAM-1 and VCAM-1 expression, as well as enhanced expression of PfEMP1 on pRBCs [64]. It potentiates pRBC sequestration through the interaction of PfEMP1 with these cell adhesion molecules. Inflammatory cytokines may also be responsible for the presence of activated microglial cells the main phagocytic macrophage-like cell population of the brain, and sequestered monocytes in CM. It has been suggested that activated myeloid cells amplify the local intra-cerebral inflammatory response - by presenting antigens to $\mathrm{T}$ cells and/or producing inflammatory cytokines. However a defined exploration of this pathway that may lead to conclusive observations in human CM is not feasible. There are significant constraints in being able to access and study important anatomical sites at crucial time points in human patients during the onset of CM symptoms. The role of $\mathrm{T}$ cells in the pathogenesis of the disease is not well documented. The existing data only provides information pertaining to the differences in the $\mathrm{T}$ cell populations in the peripheral blood of $\mathrm{CM}$ and non-CM patients, making conclusive interpretation 
of the available information very difficult [65]. Nevertheless, reductions in numbers of circulating CD4+ T cells (reflecting either sequestration in tissues or activation-induced cell death) and increased frequencies of CD4+ T cells expressing TCR Vb21.3 have been correlated with disease severity [66].

Similarly, the potential role of CD8+ T cells in mediating the observed pathology in CM has been very difficult to elucidate and there is no substantial evidence that definitively implicates the association of CD8+T cells with the development of $\mathrm{CM}$ pathogenesis. The most conclusive evidences for the involvement of the host immune responses to be associated with the precipitation of $\mathrm{CM}$ comes from studies undertaken to understand the role of genetic polymorphisms in individuals in determining the outcome of the infection [67]. The details of these associations vary from one population to another, likely reflecting differences in genetic background, but making it apparent that higher than average inflammatory responses increases the risk of development of CM. [68]. The idea that excessive pro-inflammatory immune responses pre-dispose to $\mathrm{CM}$ is also consistent with the clear age-related susceptibility to the development of $\mathrm{CM}$ in malaria endemic areas. The general observation is that very young children who are yet to acquire malaria specific cellular immune responses are relatively resistant to $\mathrm{CM}$. These individuals present themselves with severe malarial anaemia instead of developing CM symptoms. In contrast, older children who are more likely to have primed Th-1-like adaptive immune responses because of prior exposure to malaria infections are at an increased risk of developing CM Epidemiological investigations suggest that repeatedly exposed individuals eventually develop protective immunity, which may be characterized by the ability to control parasite replication by keeping parasite densities below the critical threshold for induction of hyper inflammatory immune responses to prevent $\mathrm{pRBC}$ sequestration and impede neurological damage $[68,69]$.

Experimental Cerebral Malaria: The vast majority of the immunological features of human $\mathrm{CM}$ are recapitulated during $P$. berghei ANKA infection in susceptible mice. The susceptibility of various inbred mouse strains to Experimental Cerebral Malaria (ECM) has been directly correlated with the strength of the proinflammatory immune response to the parasite and to the response of microglial and cerebral endothelial cells to these inflammatory mediators [70,71]. Experimental manipulation of $P$. berghei ANKAinfected mice has allowed causal relationships to be established between specific immune responses and the development of ECM which are consistent with the associative observations in human studies. Administration of LPS, neutralization of IL-10 or heme oxygenase 1 or inhibition of CTLA-4 signalling during $P$. berghei ANKA infection all lead to the development of ECM in normally resistant mice, whereas ablation of pro-inflammatory cytokine and chemokine signaling or depletion of macrophages prevents the development of ECM in susceptible mouse strains [72,73]. Taken together, the vast majority of experimental evidences indicate that the balance of Th- 1 to $\mathrm{T}$ regulatory responses is critical in determining the eventual outcome of these infections [74]. The manipulation of Th-2 responses in these studies did not substantially affect the outcome of infection but lowering the aggravated Th1 responses has been associated with improvement in the disease outcome [75]. Similar to humans an increase in the circulating levels of pro-inflammatory cytokines in ECM susceptible mice activates the cerebral endothelium, leading to increased expression of cell adhesion receptors, as well as upregulating chemokine production and chemokine receptor expression on leukocytes. In contrast to human cerebral malaria studies, the role of CD8+ T cells in the development of ECM in mice is well established. It has been observed that CD8 + T cells accumulate in the brains of susceptible but not resistant mice, in a CXCR3-, IP-10- (CXCL9), MIG- (CXCL10) dependent manner.

These events have been observed to occur immediately before the onset of neurological signs and are believed to directly cause disruption of the blood-brain barrier due to endothelial cell damage via perforin production [76-79]. Depletion of CD8+ T cells either from the start of infection or later during infection has been studied to completely inhibit the development of ECM. More detailed investigations indicate that $\mathrm{CD} 8+\mathrm{T}$ cells migrate to the brain in a largely antigen-specific manner, following cross-presentation of malaria antigens by classical CD8+ dendritic cells [80]. Studies performed to understand how CXCR3 upregulation on CD8+ T cells mediates migration of this cell population to the brain demonstrated that NK cell-derived IFN- $\gamma$ was required for their accumulation within the brain in susceptible mice. These observations reveal important information pertaining to the interactions between innate and adaptive immune responses in the pathogenesis of ECM, thus opening up potential new avenues of research into the role of innate immune responses, and of genetic variation in innate response genes, in the pathogenesis of human CM. In this context, effector CD4+ T cells were observed to provide help to CD8+ T cells in contributing to the development ECM as studies demonstrated that depletion of CD4+ $\mathrm{T}$ cells during the early (but not later) stages of $P$. berghei ANKA infection prevents the development of ECM [81]. In separate studies, however, depletion of CD4+ T cells during the later stages of infection also prevented the development of ECM, implying that although far fewer CD4+ cells accumulate in ECM brains in comparison to CD8+ T cells, making it apparent that CD4+ T cells may also be involved in ECM development, possibly mediating the effector phase of the disease pathogenesis. However, giventhe constraints of studying the exact sequence and interplay of different processes in humans, at present it is extremely difficult to definitively state whether the pathogenesis of CM in humans is more, or less immune mediated than what is observed in ECM in mice, and whether cells, such as CD8+ T cells, play comparable roles in the development of pathology in these two conditions. The ECM 
the model provides valuable clues to plausible events that can lead to the development of pathology during malaria infection.

\section{Adjunctive Therapies For Management of CM Pathology:} The major reasons for concern for CM in humans stem from the fact that the disease can develop rather suddenly, after just initial bouts of fever that lasts only for 2-3 days. The clinical symptoms associated with early-stage CM are not easily identifiable and is quite difficult to differentiate from encephalitis, meningitis and other febrile convulsions. This implies that the rapid and early diagnosis of the condition is often not possible, which delays the initiation of proper treatment. Coma is the standard definition of $\mathrm{CM}$, but there are other symptoms associated with the condition that include general malaise, headache, fits, vomiting and diarrhea. The early symptoms of $\mathrm{CM}$ can progress rapidly to increased intracranial pressure, hemiparesis, ataxia and coma and if immediate medical treatment is not provided it increases the risk of treatment failure and mortality. The diverse set of neurological complications that are associated with CM indicate that multiple areas of the brain are affected by the condition. Since Malaria is a complicated disease and there is no effective vaccine yet to prevent the disease, anti-malarial drug-based therapies are the only avenues for patients who present themselves with the complication of cerebral malaria. However, the incidence of neurological deficiencies and mortality remain unacceptably high with fatality rates of around 15\% following treatment with Artemisinin based combination therapies, compared with $20 \%$ for traditionally used quinine-based treatments. Given that anti-malarial therapy can only be implemented when CM is first suspected or diagnosed at health care centres; the risk from CM lies in the fact that the syndrome might already be at an advanced state in the majority of these individuals and antimalarial drugs by themselves are often insufficient to reverse and alleviate the symptoms. At present the lack of understanding of the pathogenesis of CM hinders the identification of most efficacious targets for therapeutic interventions to be properly implemented.

Therefore, there is an urgent need to develop adjunctive therapies that can be administered with anti-malarials that can, both halt the progression of $\mathrm{CM}$ and also promote healing of brain dysfunction. Based on successful case reports corticosteroids were one of the first treatments proposed as an adjunctive therapy for $\mathrm{CM}$ with the aim of reducing swelling and inflammation in the brain. However, dexamethasone failed to demonstrate a decrease in mortality in two clinical trials [82]. Similarly, treatment with intravenous immunoglobulin resulted in increased deleterious outcomes compared to the placebo group, including higher mortality and more neurological sequelae in children [83]. The failure of this therapy was attributed to not being able to reverse cytoadherence and sequestration. Curdlan Sulfate (CS), a sulfated $1 \rightarrow 3$ - $\beta$ - d glucan, a known inhibitor of $P$. falciparum in vitro, has also been tested due to its capacity to modulate the immune response to $P$. falciparum [84]. CS was expected to have some anticoagulant properties, and confer certain direct and nonspecific effect on cytoadhesion and rosetting. However the studies failed to demonstrate differences in mortality, possibly because of small sample sizes, but CS was safe and appeared to reduce the severity of the disease process [85]. Since high circulating levels of TNF has been attributed to the severity of CM pathology, therapy targeting Tumour Necrosis Factor (TNF) and its effects have also been explored. However, no difference in mortality was shown and moreover, there was an increased risk of neurological sequelae in the experimental group [86]. The failure of this therapeutic approach was thought to be due to antibody mediated retention of TNF in circulation, [86]. A variety of therapies that target a number of different mechanisms have been tested in experimental mice models. In the case of ECM, the neurologic signs appear and intensify typically after day 5 post infection (p.i.) with $P$. berghei ANKA infections, and ECM progresses rapidly after that so that after day 5 p.i., mice show BBB dysfunction, brain swelling, and hemorrhaging and necropsy. Mice begin to die by day 6 p.i., and by day 12 p.i., nearly all mice succumb to the infection. One such study in ECM tested a new formulation of glucocorticosteroid, whereby $\beta$-Methasonehemisuccinate (BMS) was encapsulated in liposomes and when administered at a late stage of infection it improved survival and prevented the development and progression of the cerebral malaria syndrome [87].

Curcumin is a non-toxic, natural anti-inflammatory molecule found in Curcuma longa rhizomes that scavenges reactive oxygen and nitrogen species [88]. In vitro studies have shown that curcumin has additive anti-parasitic activity when used in combination with artemisinins and administered in combination with arteether to mice showing symptoms of ECM. Curcumin improved survival and prevented death by lowering inflammation and impeding damage to the BBB $[88,89]$. Preclinical models have investigated the role of lithium as a potential neuroprotective intervention. Lithium has been proposed to act as a neuroprotective agent by its ability to inhibit glycogen synthase kinase 3 (GSK3 $\beta$ ), activate the PI3 K/Akt and MAPK signalling pathways, and by inducing the expression of brain-derived neurotrophic factors in neurons [90]. Lithium chloride administered to mice with ECM significantly increased the activation of Akt, which was associated with the prevention of adverse neurocognitive outcomes. Adjunctive treatment with lithium chloride was associated with better spatial and visual memory, and motor coordination in mice recovering from ECM [91]. Targeting endothelial activation and preventing microvascular permeability and vascular leak in CM can be another potential target for adjunctive therapy [92]. The angiopoietin (Ang)-Tie2 axis critically regulates endothelial cell function [93]. Perturbation of Ang-1, Ang-2 and soluble Tie2 concentrations are associated with disease severity and death in CM in both murine models and humans [94]. A mechanistic role for the Ang- Tie2 axis was established in ECM, where it was shown that Ang-1-deficient mice were more susceptible to ECM and adjunctive administration 
of a recombinant Ang-1 construct preserved BBB integrity and improved survival beyond artesunate monotherapy alone [94].

\section{Conclusion}

Malaria remains a major global health problem, associated with high morbidity and mortality. Strategies have to be developed to improve early detection and recognition of cases so that immediate treatment can be made available in order to prevent progress of the disease towards the severe condition and prevent death. For those individuals who do progress to severe forms of the disease despite prompt treatment, new tools are needed to understand how to prevent neurocognitive deterioration in CM survivors. However so far, the majority of attempts to enhance the efficacy of anti-malarial drugs with adjunctive therapy have not been very successful. The development of effective therapeutic interventions would come from a more complete understanding of the physiopathology of the disease and learning from our experiences both in humans and experimental models. Further research is needed to improve our understanding of the disease process and implement feasible strategies for effective management of CM in humans.

\section{References}

1. (2020) World Malaria Report.

2. Kantele A, Jokiranta S (2010) Plasmodium knowlesi--the fifth species causing human malaria. Duodecim 126(4): 427-434.

3. Sierro F, Grau GER (2019) The Ins and Outs of Cerebral Malaria Pathogenesis: Immunopathology, Extracellular Vesicles, Immunometabolism, and Trained Immunity. Front Immunol 10: 830.

4. Idro R, Ndiritu M, Ogutu B, Mithwani S, Maitland K, et al. (2007) Burden, features, and outcome of neurological involvement in acute falciparum malaria in Kenyan children. JAMA 297(20): 2232-2240.

5. Postels DG, Birbeck GL (2000) Severe falciparum malaria. World Health Organization, Communicable Diseases Cluster. Trans R Soc Trop Med Hyg 94: S1-S90.

6. Hochman SE, Madaline TF, Wassmer SC, Mbale E, Choi N, et al. (2015) Fatal Pediatric Cerebral Malaria Is Associated with Intravascular Monocytes and Platelets That Are Increased with HIV Coinfection mBio 6(5): e01390-e01415.

7. John CC, Bangirana P, Byarugaba J, Opoka RO, Idro R, et al. (2018) Cerebral malaria in children is associated with long-term cognitive impairment. Pediatrics 122(1): e92-e99.

8. Crompton PD, Moebius J, Portugal S, Waisberg M, Hart G, et al. (2014) Malaria immunity in man and mosquito: insights into unsolved mysteries of a deadly infectious disease. Annu Rev Immunol 32: 157-187.

9. Fowkes FJ, Boeuf P, Beeson JG (2016) Immunity to malaria in an era of declining malaria transmission. Parasitology 143(2): 139-153.

10. Artavanis-Tsakonas K, Tongren JE, Riley EM (2003) The war between the malaria parasite and the immune system: immunity, immunoregulation and immunopathology Clin Exp Immunol 133(2): 145-152.

11. Gonçalves RM, Lima NF, Ferreira MU (2014) Parasite virulence, coinfections and cytokine balance in malaria. Pathog Glob Health 108(4): 173-178.

12. Deroost K, Pham TT, Opdenakker G, Van den Steen PE (2016) The immunological balance between host and parasite in malaria. FEMS Microbiol Rev 40(2): 208-257.
13. Haldar K, Murphy SC, Milner DA, Taylor TE (2007) Malaria: mechanisms of erythrocytic infection and pathological correlates of severe disease. Annu Rev Pathol 2: 217-249.

14. Patnaik JK, Das BS, Mishra SK, Mohanty S, Satpathy SK, et al. (1994) Vascular clogging, mononuclear cell margination, and enhanced vascular permeability in the pathogenesis of human cerebral malaria. Am J Trop Med Hyg 51(5): 642-647.

15. Mishra SK, Wiese L (2009) Advances in the management of cerebral malaria in adults. Curr Opin Neurol 22(3): 302-307.

16. Clark IA, Awburn MM, Whitten RO, Harper CG, Liomba NG, et al. (2003) Tissue distribution of migration inhibitory factor and inducible nitric oxide synthase in falciparum malaria and sepsis in African children. Malar J 2: 6.

17. Taylor TE, Fu WJ, Carr RA, Whitten RO, Mueller JS, et al. (2004) Differentiating the pathologies of cerebral malaria by postmortem parasite counts. Nat Med 10(2): 143-145.

18. Newbold C, Craig A, Kyes S, Rowe A, Fernandez-Reyes D, et al. (1999) Cytoadherence, pathogenesis and the infected red cell surface in Plasmodium falciparum. Int J Parasitol 29(6): 927-937.

19. Bate CA, Kwiatkowski DP (1999) Stimulators of tumour necrosis factor production released by damaged erythrocytes. Immunology 83(2): 256261.

20. Schofield L, Novakovic S, Gerold P, Schwarz RT, McConville MJ, et al. (1996) Glycosylphosphatidylinositol toxin of Plasmodium up-regulates intercellular adhesion molecule-1, vascular cell adhesion molecule-1, and E-selectin expression in vascular endothelial cells and increases leukocyte and parasite cytoadherence via tyrosine kinase-dependent signal transduction. J Immunol 156(5): 1886-1896.

21. Penet MF, Kober F, Confort-Gouny S, Le Fur Y, Dalmasso C, et al. (2007) Magnetic resonance spectroscopy reveals an impaired brain metabolic profile in mice resistant to cerebral malaria infected with Plasmodium berghei ANKA. J Biol Chem 282(19): 14505-14514.

22. Penet MF, Viola A, Confort-Gouny S, Le Fur Y, Duhamel G, et al. (2005) Imaging experimental cerebral malaria in vivo: significant role of ischemic brain edema. J Neurosci 25(32): 7352-7358.

23. Silamut K, Phu NH, Whitty C, Turner GD, Louwrier K, et al. (1999) A quantitative analysis of the microvascular sequestration of malaria parasites in the human brain. Am J Pathol 155(2): 395-410.

24. Dondorp AM, Desakorn V, Pongtavornpinyo W, Sahassananda D, Silamut K, et al. (2005) Estimation of the total parasite biomass in acute falciparum malaria from plasma PfHRP2. PLoS Med 2(8): e204.

25. Hau J, Van Hoosier GL Jr (2005) Handbook of Laboratory Animal Science ( $2^{\text {nd }}$ Edn.)., CRC Press, Boca Raton, FL, USA.

26. Aikawa M, Brown A, Smith CD, Tegoshi T, Howard RJ, et al. (1992) A primate model for human cerebral malaria: Plasmodium coatneyiinfected rhesus monkeys. Am J Trop Med Hyg 46(4): 391-397.

27. Gysin J, Aikawa M, Tourneur N, Tegoshi T (1992) Experimental Plasmodium falciparum cerebral malaria in the squirrel monkey Saimirisciureus. Exp Parasitol 75(4): 390-398.

28. Rest JR (1982) Cerebral malaria in inbred mice. I. A new model and its pathology. Trans R Soc Trop Med Hyg 76(3): 410-415.

29. Curfs JH, Van der Meide PH, Billiau A, Meuwissen JH, Eling WM (1993) Plasmodium berghei: recombinant interferon-gamma and the development of parasitemia and cerebral lesions in malaria-infected mice. Exp Parasitol 77(2): 212-223.

30. Yoeli M, Hargreaves BJ (1974) Brain capillary blockage produced by a virulent strain of rodent malaria. Science 184(4136): 572-573.

31. Couper KN, Blount DG, Hafalla JC, Van Rooijen N, De Souza JB, et al. (2007) Macrophage-mediated but gamma interferon-independent 
innate immune responses control the primary wave of Plasmodium yoeliiparasitemia. Infect Immun 75(12): 5806-5818.

32. Couper KN, Blount DG, Wilson MS, Hafalla JC, Belkaid Y, et al. (2008) IL10 from CD4CD25Foxp3CD127 adaptive regulatory $\mathrm{T}$ cells modulates parasite clearance and pathology during malaria infection. PLoS Pathog 4(2): e1000004.

33. Kaul DK, Nagel RL, Llena JF, Shear HL (1994) Cerebral malaria in mice: demonstration of cytoadherence of infected red blood cells and microrheologic correlates. Am J Trop Med Hyg 50(4): 512-521.

34. Curfs JH, Hermsen CC, Kremsner P, Neifer S, Meuwissen JH, et al. (1993) Tumour necrosis factor-alpha and macrophages in Plasmodium bergheiinduced cerebral malaria. Parasitology 107(2): 125-134.

35. Mitchell AJ, Hansen AM, Hee L, Ball HJ, Potter SM, et al. (2005) Early cytokine production is associated with protection from murine cerebral malaria. Infect Immun 73(9): 5645-5653.

36. De Souza JB, Riley EM (2002) Cerebral malaria: the contribution of studies in animal models to our understanding of immunopathogenesis. Microbes Infect 4(3): 291-300.

37. Clark CJ, Phillips RS, McMillan RB, Montgomery IO, Stone TW (2005) Differences in the neurochemical characteristics of the cortex and striatum of mice with cerebral malaria. Parasitology 130(1): 23-29.

38. Engwerda CR, Mynott TL, Sawhney S, De Souza JB, Bickle QD, et al. (2002) Locally up-regulated lymphotoxin alpha, not systemic tumor necrosis factor alpha, is the principle mediator of murine cerebral malaria. J Exp Med 195(10): 1371-1317.

39. Hearn J, Rayment N, Landon DN, Katz DR, De Souza JB (2000) Immunopathology of cerebral malaria: morphological evidence of parasite sequestration in murine brain microvasculature. Infect Immun 68(9): 5364-5376.

40. Wassmer SC, Combes V, Grau GE (2003) Pathophysiology of cerebra malaria: role of host cells in the modulation of cytoadhesion. Ann N Y Acad Sci 992: 30-38.

41. Von ZurMuhlen C, Sibson NR, Peter K, Campbell SJ, Wilainam P, et al (2008) A contrast agent recognizing activated platelets reveals murine cerebral malaria pathology undetectable by conventional MRI. J Clin Invest 118(3): 1198-1207.

42. Desruisseaux MS, Gulinello M, Smith DN, Lee SC, Tsuji M, et al. (2008) Cognitive dysfunction in mice infected with Plasmodium berghei strain ANKA. J Infect Dis 197(11): 1621-1627.

43. Greiner J, Dorovini-Zis K, Taylor TE, Molyneux ME, Beare NA, et al. (2015) Correlation of hemorrhage, axonal damage, and blood-tissue barrier disruption in brain and retina of Malawian children with fatal cerebral malaria. Front Cell Infect Microbiol 5: 18.

44. Seydel KB, Kampondeni SD, Valim C, Potchen MJ, Milner DA, et al. (2015) Brain swelling and death in children with cerebral malaria. N Engl J Med 372(12): 1126-1137.

45. Mohanty S, Benjamin LA, Majhi M, Panda P, Kampondeni S, et al. (2017) Magnetic Resonance Imaging of Cerebral Malaria Patients Reveals Distinct Pathogenetic Processes in Different Parts of the Brain. M Sphere 2(3): e00193-e00217.

46. Potchen MJ, Kampondeni SD, Seydel KB, Haacke EM, Sinyangwe SS, et al (2018) 1.5 Tesla Magnetic Resonance Imaging to Investigate Potential Etiologies of Brain Swelling in Pediatric Cerebral Malaria. Am J Trop Med Hyg 98(2): 497-504.

47. Daneman R, Prat A (2015) The blood-brain barrier. Cold Spring Harb Perspect Biol 7(1): a020412.

48. Storm J, Craig AG (2014) Pathogenesis of cerebral malaria--inflammation and cytoadherence. Front Cell Infect Microbiol 4: 100.

49. Dunst J, Kamena F, Matuschewski K (2017) Cytokines and Chemokines in Cerebral Malaria Pathogenesis. Front Cell Infect Microbiol 7: 324
50. Moxon CA, Wassmer SC, Milner DA Jr, Chisala NV, Taylor TE, et al. (2013) Loss of endothelial protein $C$ receptors links coagulation and inflammation to parasite sequestration in cerebral malaria in African children. Blood 122(5): 842-851.

51. Brussee JM, Yeo TW, Lampah DA, Anstey NM, Duffull SB (2015) Pharmacokinetic-Pharmacodynamic Model for the Effect of l-Arginine on Endothelial Function in Patients with Moderately Severe Falciparum Malaria. Antimicrob Agents Chemother 60(1): 198-205.

52. Rug M, Prescott SW, Fernandez KM, Cooke BM, Cowman AF (2006) The role of KAHRP domains in knob formation and cytoadherence of $\mathrm{P}$ falciparum-infected human erythrocytes. Blood 108(1): 370-378.

53. Rowe JA, Claessens A, Corrigan RA, Arman M (2009) Adhesion of Plasmodium falciparum-infected erythrocytes to human cells: molecular mechanisms and therapeutic implications. Expert Rev Mol Med 11: e16.

54. Lennartz F, Adams Y, Bengtsson A, Olsen RW, Turner L, et al. (2017) Structure-Guided Identification of a Family of Dual Receptor-Binding PfEMP1 that Is Associated with Cerebral Malaria. Cell Host Microbe 21(3): 403-414.

55. Avril M, Bernabeu M, Benjamin M, Brazier AJ, Smith JD (2016) Interaction between Endothelial Protein C Receptor and Intercellular Adhesion Molecule 1 to Mediate Binding of Plasmodium falciparum-Infected Erythrocytes to Endothelial Cells. mBio 7(4): e00615-e00616.

56. Kessler A, Dankwa S, Bernabeu M, Harawa V, Danziger SA, et al. (2017) Linking EPCR-Binding PfEMP1 to Brain Swelling in Pediatric Cerebral Malaria. Cell Host Microbe 22(5): 601-614.

57. Storm J, Jespersen JS, Seydel KB, Szestak T, Mbewe M, et al. (2019) Cerebral malaria is associated with differential cytoadherence to brain endothelial cells. EMBO Mol Med 11(2): e9164.

58. Chan JA, Howell KB, Reiling L, Ataide R, Mackintosh CL, et al. (2012) Targets of antibodies against Plasmodium falciparum-infected erythrocytes in malaria immunity. J Clin Invest 122(9): 3227-3238.

59. Pain A, Ferguson DJ, Kai O, Urban BC, Lowe B, et al. (2001) Plateletmediated clumping of Plasmodium falciparum-infected erythrocytes is a common adhesive phenotype and is associated with severe malaria. Proc Natl AcadSci U S A 98(4): 1805-1810.

60. Wang CW, Hviid L (2015) Rifins, rosetting, and red blood cells. Trends Parasitol 31(7): 285-286.

61. Glennon EKK, Dankwa S, Smith JD, Kaushansky A (2018) Opportunities for Host-targeted Therapies for Malaria. Trends Parasitol 34(10): 843860 .

62. Wittchen ES (2009) Endothelial signaling in paracellular and transcellular leukocyte transmigration. Front Biosci (Landmark Ed) 14: 2522-2545.

63. John CC, Panoskaltsis-Mortari A, Opoka RO, Park GS, Orchard PJ, et al. (2008) Cerebrospinal fluid cytokine levels and cognitive impairment in cerebral malaria. Am J Trop Med Hyg 78(2): 198-205.

64. Tripathi AK, Sha W, Shulaev V, Stins MF, Sullivan DJ Jr (2009) Plasmodium falciparum-infected erythrocytes induce NF-kappaB regulated inflammatory pathways in human cerebral endothelium. Blood 114(19): 4243-4252.

65. Udomsangpetch R, Pipitaporn B, Silamut K, Pinches R, Kyes S, et al. (2002) Febrile temperatures induce cytoadherence of ring-stage Plasmodium falciparum-infected erythrocytes. Proc Natl AcadSci U S A 99(18): 11825-11829.

66. Elhassan IM, Hviid L, Satti G, Akerstrom B, Jakobsen PH, et al. (1994) Evidence of endothelial inflammation, $\mathrm{T}$ cell activation, and $\mathrm{T}$ cell reallocation in uncomplicated Plasmodium falciparum malaria. Am J Trop Med Hyg 51(3): 372-379.

67. Loizon S, Boeuf P, Tetteh JK, Goka B, Obeng-Adjei G, et al. (2007) V beta profiles in African children with acute cerebral or uncomplicated 
malaria: very focused changes among a remarkable global stability. Microbes Infect 9(11): 1252-1259.

68. Verra F, Mangano VD, Modiano D (2009) Genetics of susceptibility to Plasmodium falciparum: from classical malaria resistance genes towards genome-wide association studies. Parasite Immunol 31(5): 234-253.

69. Walther M, Jeffries D, Finney OC, Njie M, Ebonyi A, et al. (2009) Distinct roles for FOXP3 and FOXP3 CD4 T cells in regulating cellular immunity to uncomplicated and severe Plasmodium falciparum malaria. PLoS Pathog 5(4): e1000364.

70. Lou J, Gasche Y, Zheng L, Critico B, Monso-Hinard C, et al. (1998) Differential reactivity of brain microvascular endothelial cells to TNF reflects the genetic susceptibility to cerebral malaria. Eur J Immunol 28(12): 3989-4000.

71. Randall LM, Amante FH, Mc Sweeney KA, Zhou Y, Stanley AC, et al. (2008) Common strategies to prevent and modulate experimental cerebral malaria in mouse strains with different susceptibilities. Infect Immun 76(7): 3312-3320

72. Kossodo S, Monso C, Juillard P, Velu T, Goldman M, et al. (1997) Interleukin-10 modulates susceptibility in experimental cerebral malaria. Immunology 91(4): 536-540.

73. Neill AL, Hunt NH (1995) Effects of endotoxin and dexamethasone on cerebral malaria in mice. Parasitology 11(4): 443-454.

74. Nie CQ Bernard NJ, Schofield L, Hansen DS (2007) CD4+ CD25+ regulatory $\mathrm{T}$ cells suppress CD4+ T-cell function and inhibit the development of Plasmodium berghei-specific TH1 responses involved in cerebral malaria pathogenesis. Infect Immun 75(5): 2275-2282.

75. Saeftel M, Krueger A, Arriens S, Heussler V, Racz P, et al. (2004) Mice deficient in interleukin-4 (IL-4) or IL-4 receptor alpha have higher resistance to sporozoite infection with Plasmodium berghei (ANKA) than do naive wild-type mice. Infect Immun 72(1): 322-331.

76. Rénia L, Potter SM, Mauduit M, Rosa DS, Kayibanda M, et al. (2006) Pathogenic T cells in cerebral malaria. Int J Parasitol 36(5): 547-554.

77. Howland SW, Claser C, Poh CM, Gun SY, Rénia L (2015) Pathogenic CD8+ $\mathrm{T}$ cells in experimental cerebral malaria. Semin Immunopathol 37(3): 221-231.

78. Potter S, Chan-Ling T, Ball HJ, Mansour H, Mitchell A, et al. (2006) Perforin mediated apoptosis of cerebral microvascular endothelial cells during experimental cerebral malaria. Int J Parasitol 36(4): 485-496.

79. Belnoue E, Kayibanda M, Vigario AM, Deschemin JC, Van Rooijen N, et al. (2002) On the pathogenic role of brain-sequestered alphabeta CD8+ T cells in experimental cerebral malaria. J Immunol 169(11): 6369-6375.

80. Lundie RJ, De Koning-Ward TF, Davey GM, Nie CQ, Hansen DS, et al. (2008) Blood-stage Plasmodium infection induces CD8+ T lymphocytes to parasite-expressed antigens, largely regulated by CD8alpha+ dendritic cells. Proc Natl AcadSci U S A 105(38): 14509-14514.

81. Good MF, Xu H, Wykes M, Engwerda CR (2005) Development and regulation of cell-mediated immune responses to the blood stages of

\section{ISSN: 2574-1241}

DOI: $10.26717 /$ BJSTR.2021.35.005639

\section{Santosh K Kar. Biomed J Sci \& Tech Res}

This work is licensed under Creative Commons Attribution 4.0 License

Submission Link: https://biomedres.us/submit-manuscript.php malaria: implications for vaccine research. Annu Rev Immunol 23: 6999.

82. Warrell DA, Looareesuwan S, Warrell MJ, Kasemsarn P, Intaraprasert R, et al. (1982) Dexamethasone proves deleterious in cerebral malaria. A double-blind trial in 100 comatose patients. N Engl J Med 306(6): 313319.

83. Taylor TE, Molyneux ME, Wirima JJ, Borgstein A, Goldring JD, et al. (1992) Intravenous immunoglobulin in the treatment of paediatric cerebral malaria. ClinExpImmunol 90(3): 357-362.

84. Havlik I, Rovelli S, Kaneko Y (1994) The effect of curdlansulphate on in vitro growth of Plasmodium falciparum. Trans R Soc Trop Med Hyg 88(6): 686-687.

85. Havlik I, Looareesuwan S, Vannaphan S, Wilairatana P, Krudsood S, et al. (2005) Curdlansulphate in human severe/cerebral Plasmodium falciparum malaria. Trans R Soc Trop Med Hyg 99(5): 333-340.

86. VanHensbroek MB, Palmer A, Onyiorah E, Schneider G, Jaffar S, et al. (1996) The effect of a monoclonal antibody to tumor necrosis factor on survival from childhood cerebral malaria. J Infect Dis 174(5): 10911097.

87. Waknine-Grinberg JH, Even-Chen S, Avichzer J, Turjeman K, BenturaMarciano A, et al. (2013) Glucocorticosteroidsin nano-sterically stabilized liposomes are efficacious for elimination of the acute symptoms of experimental cerebral malaria. PLoS One 8(8): e72722.

88. Reddy RC, Vatsala PG, Keshamouni VG, Padmanaban G, Rangarajan PN (2005) Curcumin for malaria therapy. BiochemBiophys Res Commun 326(2): 472-474

89. Dende C, Meena J, Nagarajan P, Panda AK, Rangarajan PN, et al. (2015) Simultaneously targeting inflammatory response and parasite sequestration in brain to treat Experimental Cerebral Malaria Sci Rep 5: 12671

90. Rowe MK, Chuang DM (2004) Lithium neuroprotection: molecular mechanisms and clinical implications. Expert Rev Mol Med 6(21): 1-18.

91. Dai M, Freeman B, Shikani HJ, Bruno FP, Collado JE, et al. (2012) Altered regulation of Akt signaling with murine cerebral malaria, effects on longterm neuro-cognitive function, restoration with lithium treatment. PLoS One 7(10): e44117.

92. Kim H, Higgins S, Liles WC, Kain KC (2011) Endothelial activation and dysregulation in malaria: a potential target for novel therapeutics. Curr Opin Hematol 18(3): 177-185.

93. Augustin HG, Koh GY, Thurston G, Alitalo K (2009) Control of vascular morphogenesis and homeostasis through the angiopoietin-Tie system. Nat Rev Mol Cell Biol 10(3): 165-177.

94. Higgins SJ, Purcell LA, Silver KL, Tran V, Crowley V, et al. (2016) Dysregulation of angiopoietin-1 plays a mechanistic role in the pathogenesis of cerebral malaria. Sci Transl Med 8(358): 358ra128.

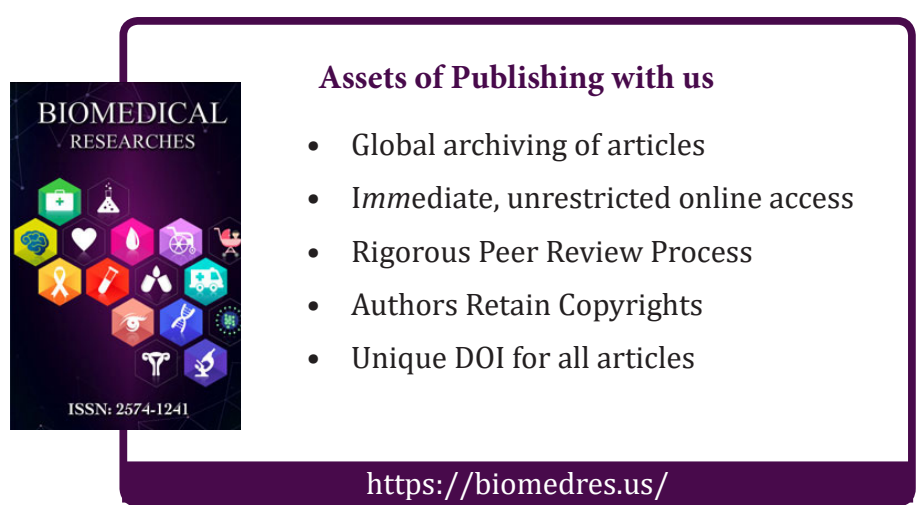

